\title{
String tension between de Sitter vacua and curvature corrections
}

\author{
Ivano Basile $\circledast^{1, *}$ and Alessia Platania $\odot^{2, \dagger}$ \\ ${ }^{1}$ Service de Physique de l'Univers, Champs et Gravitation, Université de Mons, \\ Place du Parc 20, 7000 Mons, Belgium \\ ${ }^{2}$ Perimeter Institute for Theoretical Physics, 31 Caroline Street North, Waterloo, Ontario N2L 2Y5, Canada
}

(Received 24 March 2021; accepted 19 November 2021; published 6 December 2021)

\begin{abstract}
Higher-derivative corrections to cosmological effective actions in string theory are largely constrained by T-duality, but have been computed hitherto only to the first few orders in the string scale $\alpha^{\prime}$. The functional renormalization group, in conjunction with the strong constraints imposed by T-duality, allows us to derive cosmological effective actions to all orders in $\alpha^{\prime}$ while avoiding "truncations" of the theory space. We show that the resulting higher-derivative $\alpha^{\prime}$-corrections forbid the existence of de Sitter vacua, at least in regimes where string-loop corrections can be neglected. Our findings thus support the no-de Sitter swampland conjecture in the presence of all-order effects in $\alpha^{\prime}$.
\end{abstract}

DOI: 10.1103/PhysRevD.104.L121901

\section{INTRODUCTION}

Effective actions in quantum field theories (QFTs) embody quantum fluctuations on all scales in a single functional of the degrees of freedom of the theory. Their knowledge is thus paramount to shed light on a variety of phenomena that could be compared with observations. Whenever a field theory remains weakly coupled at all scales, perturbative methods generally provide adequate computational control. However, in the case of gravity, all-order (and possibly nonperturbative) effects appear necessary to capture all of the relevant physics.

In asymptotically safe gravity [1-4] these effects ought to arise from a non-Gaussian ultraviolet fixed point, whose existence would make the theory "asymptotically safe," i.e., nonperturbatively renormalizable in the Wilsonian sense [1,2]. In (perturbative) string theory, these effects arise from a double expansion involving the string scale $\alpha^{\prime}$ and the string coupling $g_{s}$. Although well defined in principle, the computation in general settings is highly involved, and all-order computations in the absence of supersymmetry can be carried out only in specific backgrounds, such as the linear dilaton background and the ones studied in [5,6].

In contrast, phenomenologically relevant settings, such as de Sitter (dS) vacua for both early- and late-time cosmology,

\footnotetext{
*ivano.basile@umons.ac.be

aplatania@perimeterinstitute.ca
}

Published by the American Physical Society under the terms of the Creative Commons Attribution 4.0 International license. Further distribution of this work must maintain attribution to the author(s) and the published article's title, journal citation, and DOI. Funded by SCOAP . have proven much subtler since the supersymmetry algebra cannot be (linearly) realized in $\mathrm{dS}$, and their existence in string theory remains under detailed scrutiny [7-15]. Some supersymmetry-breaking mechanisms appear to naturally yield other types of cosmologies [16], whose implications for cosmic microwave background anomalies have been explored in [17-19], while the associated instabilities suggest the possibility of dS braneworlds [20]. At present, however, the consistency of a number of $\mathrm{dS}$ constructions is unsettled, and a deeper understanding could require the inclusion of all-order effects.

In an attempt to shed some light on whether dS vacua are allowed in the presence of all-order effects, in this paper we employ functional renormalization group (FRG) techniques [21] to resum $\alpha^{\prime}$ corrections and investigate the resulting string cosmologies. In the context of the FRG, the object of interest is the effective average action (EAA) [22] since its flow allows us, at least in principle, to infer the existence of an asymptotically safe regime, granting nonperturbative renormalizability [1], and/or to recover the exact quantum effective action via its RG flow in the IR. One of the major practical limitations of this approach is typically the need to truncate the theory space in order to feasibly solve the flow equations [21]. However, the remarkable constraints that string theory entails on its low-energy theory space, which arises from integrating out its higher-spin massive modes, may partially circumvent this issue. Concretely, in cosmological settings T-duality constrains the minisuperspace ${ }^{1}$

\footnotetext{
${ }^{1}$ Despite its limitations, the minisuperspace approach has proven useful in quantum cosmology, as for instance in [23-27]. In the present setting, it allows us to exploit the classification of [28-31] in order to obtain all-order curvature corrections [32].
} 
effective action to a large extent $[28-31,33,34]^{2}$ and could therefore go hand in hand with FRG methods to capture all-order effects in $\alpha^{\prime}$. In this paper we provide a proof of principle of this idea, applying the FRG to resum $\alpha^{\prime}$ corrections to all orders in cosmological backgrounds, neglecting $g_{s}$ string loop corrections. Based on two closed-form solutions to the (minisuperspace) flow equations, we show that $\mathrm{dS}$ vacua are not compatible with the resulting quantum-corrected cosmologies, thus providing support for the no-dS swampland conjecture [35-38] and highlighting a tension between de Sitter vacua and curvature corrections in string theory.

\section{EFFECTIVE ACTIONS AND COSMOLOGY}

The existence of $\mathrm{dS}$ solutions is a relevant open question in quantum gravity, in particular, within the context of string theory and the swampland program at large [10]. Whether they can be realized relies crucially on the form of the gravitational quantum effective action. In any metric approach to quantum gravity based on QFT (e.g., quantum unimodular gravity, asymptotically safe gravity, Hořava-Lifshitz gravity, etc.), if diffeomorphism invariance holds at high energies and no additional symmetries constraining the gravitational interaction arise, the Einstein-Hilbert term ought to be complemented by all possible curvature invariants. Deriving the effective action exactly, at least within a minisuperspace scheme [39-43], requires summing over all quantum fluctuations at the level of a gravitational functional integral or, equivalently, solving the FRG equations [21]. While these computations are generally unfeasible in the untruncated space of diffeomorphism-invariant theories (even in minisuperspace), the additional symmetries of string theory make it an ideal candidate in which to determine cosmological effective actions with FRG methods [32] and investigate the existence of dS solutions.

Gravitational effective actions in string theory can be derived, at least at leading order in the string coupling $g_{s}$, from the world-sheet formulation, and their form in cosmological backgrounds is strongly constrained by T-duality. Specifically, in $D=d+1$ dimensions, T-duality emerges as an $O(d, d, \mathbb{R})$ symmetry on the low-energy degrees of freedom [28-30], namely, the dilaton $\phi$, the metric $G$, and the Kalb-Ramond two-form $B_{2}$. Focusing on cosmological backgrounds of the type

$$
\begin{aligned}
d s^{2} & =-n^{2}(t) d t^{2}+e^{2 \sigma(t)} d \mathbf{x}^{2}, \\
B_{2} & =0, \quad \Phi=\Phi(t),
\end{aligned}
$$

\footnotetext{
${ }^{2}$ The swampland program also purports constraining the lowenergy theory space from the high-energy vantage point of string theory $[10,35]$ and could potentially further simplify FRG computations.
}

where we have defined $\Phi(t)$ in terms of $\phi$ as in $[29-31,33,34]$, the tree-level action reduces to

$$
S_{\mathrm{red}}=\frac{\mathrm{Vol}_{d}}{16 \pi G_{\mathrm{N}}} \int d t \frac{1}{n} e^{-\Phi}\left(-\dot{\Phi}^{2}+d \dot{\sigma}^{2}\right),
$$

where $G_{\mathrm{N}}$ is the $D$-dimensional Newton constant, $\mathrm{Vol}_{d}$ is the volume of $d$-dimensional spatial slices, and $H=\dot{\sigma}$ is the Hubble parameter. All $\alpha^{\prime}$-corrections are encoded in the higher-derivative part of the Meissner-Hohm-Zwiebach effective action [29-31,33,34]. On cosmological backgrounds, T-duality requires that the effective action be even in $H$. Specifically, it can be shown [29-31,33,34] that its all-order expression reads ${ }^{3}$

$$
\begin{aligned}
S_{\mathrm{HD}} \sim & \frac{\mathrm{Vol}_{d}}{16 \pi G_{\mathrm{N}}} \int d t \frac{e^{-\Phi}}{n} \\
& \times\left[-\dot{\Phi}^{2}+2 d n^{2} \sum_{m=0}^{\infty}(-4)^{m} c_{m} \alpha^{\prime m-1}\left(\frac{H}{n}\right)^{2 m}\right] .
\end{aligned}
$$

In string perturbation theory, obtaining the coefficients $c_{m}$ is typically a daunting task, even for low-order coefficients. In this work, we shall attempt to overcome these issues and compute all the $c_{m}$ at once via FRG techniques [21]. The latter allow one to determine the flow of the EAA $\Gamma_{k}$ via the exact flow equation $[22,44,45]$

$$
k \partial_{k} \Gamma_{k}=\frac{1}{2} \operatorname{STr}\left\{\left(\Gamma_{k}^{(2)}+\mathcal{R}_{k}\right)^{-1} k \partial_{k} \mathcal{R}_{k}\right\} .
$$

The supertrace on the right-hand side denotes a sum over internal indices and an integral over continuum spacetime coordinates or momenta. ${ }^{4}$ The function $\mathcal{R}_{k}$ is a regulator which implements the Wilsonian shell-by-shell integration of fluctuating modes: At a scale $k$, all quantum fluctuations with momenta $p^{2}>k^{2}$ are integrated out, and the quantum effective action ought to be recovered in the IR limit $k \rightarrow 0$. To wit, the regulator $\mathcal{R}_{k}$ has to vanish as $k \rightarrow 0$, where indeed $\Gamma_{k \rightarrow 0}$ reduces to the standard quantum effective action. On the other hand, a UV-complete theory ought to flow to a fixed point as $k \rightarrow \infty$, while trajectories off the UV critical surface correspond to theories which are inconsistent and thus belong to the "swampland," as shown in Fig. 1. Let us also remark that nonlocality, which is an expected feature of quantum gravity, can be treated within the FRG (see [46] for examples in the context of asymptotically safe gravity). Specifically, the formalism could, in principle, account for

\footnotetext{
${ }^{3}$ Note that the effective action in Eq. (3) is only valid for $g_{s}=e^{\phi} \ll 1$, whereby string-loop corrections are expected to be negligible. In this limit, T-duality is indeed realized as a continuous $O(d, d, \mathbb{R})$ symmetry, whereas $g_{s}$-corrections generally break it to $O(d, d, \mathbb{Z})$.

${ }^{4}$ Let us remark that functional traces ought to be defined via the inner product induced by the kinetic terms of the classical action.
} 


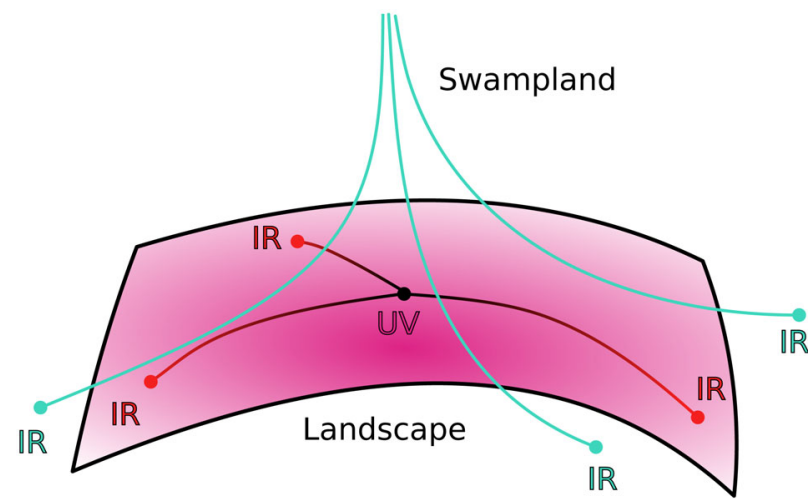

FIG. 1. Pictorial representation of the "landscape" of lowenergy theories which admit a UV completion as a UV critical surface. The trajectories off the critical surface (green curves) belong to the "swampland" of inconsistent theories.

UV/IR mixing by constraining the IR physics via nontrivial requirements stemming from UV completeness.

Since T-duality imposes strong constraints on the form of the effective action in cosmological backgrounds, the flow of a scale-dependent Meissner-Hohm-Zwiebach EAA can be computed analytically, avoiding truncations of the theory space. ${ }^{5}$ To this end, the first step is to promote all couplings in Eq. (3) to scale-dependent quantities

$$
c_{m} \rightarrow c_{m}(k), \quad G_{\mathrm{N}} \rightarrow G_{k} \equiv g_{k} \frac{k^{1-d}}{16 \pi},
$$

where the factor of $16 \pi$ is introduced for convenience. All $\alpha^{\prime}$-corrections are thus conveniently encoded in an even, dimensionless, scale-dependent function $F_{k}$, according to

$\Gamma_{k}=\operatorname{Vol}_{d} \int d t \frac{e^{-\Phi}}{n}\left[-\frac{k^{d-1}}{g_{k}} \dot{\Phi}^{2}+k^{d+1} n^{2} F_{k}\left(\frac{H^{2}}{n^{2}}\right)\right]$.

In what follows we shall gauge-fix $n=1$, so that ghosts trivially decouple.

Given a solution $\Gamma_{k}$, one can send $k \rightarrow 0$ and extract the corresponding coefficients $c_{m} \equiv c_{m}(0)$. This procedure would then yield $\alpha^{\prime}$-corrections for the MeissnerHohm-Zwiebach action [29-31,33,34] to all orders, a task that would appear, at present, out of reach in perturbative string theory. In addition, the effective action $\Gamma_{0}$ gives access to a number of observable quantities and allows one, at least in principle, to compute solutions to the quantum-corrected field equations and address a

\footnotetext{
${ }^{5}$ Although T-duality constrains the effective action, the presence of a regulator could induce deviations from the hypersurface of T-duality-invariant theories. Nevertheless, since T-duality ought to reemerge in the limit $k \rightarrow 0$ [30,31,33,34], we expect these deviations to be small, and we neglect T-duality-breaking terms.
}

number of interesting challenges, such as the puzzling apparent lack of dS solutions [36-38].

\section{EXISTENCE OF DE SITTER VACUA}

Resumming $\alpha^{\prime}$-corrections to all orders entails solving Eq. (4) for the scale-dependent Meissner-Hohm-Zwiebach effective action in Eq. (6). To this end, one has to derive the beta functions for the coupling $g_{k}$ and the function $F_{k}$. This amounts to replacing the scale-dependent MeissnerHohm-Zwiebach effective action of Eq. (6) in Eq. (4). The flow equations for $g_{k}$ and $F_{k}\left(H^{2}\right)$ have been derived in [32]. They admit a particular solution for any $d$ given by

$$
\begin{gathered}
g_{k}=\frac{g_{0} g_{*}}{g_{*} k_{0}^{d-1}+g_{0}\left(k^{d-1}-k_{0}^{d-1}\right)} k^{d-1}, \\
F_{k}\left(H^{2}\right)=k^{-d-1}\left(c_{0}+c_{1} \sqrt{H^{2}}\right),
\end{gathered}
$$

where $c_{0}, c_{1}$, and $g_{0}$ are integration constants, $k_{0}$ is a reference scale, and $g_{*}=2^{d} d(d-1) \pi^{1+\frac{d}{2}} \Gamma\left(\frac{d}{2}\right)$ is the position of the non-Gaussian UV fixed point for $g_{k}$. Interestingly, despite T-duality, the running of the Newton coupling in Eq. (7) matches the one found in asymptotically safe gravity [47]. This suggestive result is nontrivially consistent with our scenario, where the string effective action arises in the IR from a UV fixed-point action that is closely approached by the RG trajectory of string theory. In particular, this resonates with the intriguing possibility that asymptotically safe gravity sits in a corner of the theory space of string theory [48], thus realizing a scenario similar to that advocated by Weinberg in [49]. ${ }^{6}$ The resulting effective action reads

$$
\Gamma_{\text {string }}=\frac{\mathrm{Vol}_{d}}{16 \pi G_{\mathrm{N}}} \int d t n e^{-\Phi}\left[-\frac{\dot{\Phi}^{2}}{n^{2}}+\left(\Lambda+\tilde{c} \sqrt{\frac{H^{2}}{n^{2} \Lambda}}\right)\right],
$$

where we have restored the lapse $n$, we have introduced the effective coupling $\tilde{c}=\frac{\sqrt{\Lambda}}{16 \pi G_{\mathrm{N}}} c_{1}$, and we have identified $c_{0} \equiv \frac{\Lambda}{16 \pi G_{\mathrm{N}}}$, where $\Lambda \approx 1 / \alpha^{\prime}$ is the (leading contribution to the) string-frame cosmological constant. ${ }^{7}$ The corresponding cosmological field equations take the simple form

$$
\dot{\Phi}^{2}=-\Lambda, \quad \ddot{\Phi}=\frac{\tilde{c}}{2} \sqrt{\frac{H^{2}}{\Lambda}}, \quad \frac{\tilde{c}}{\Lambda^{2}} \frac{|H|}{H} \dot{\Phi}=0,
$$

and it is straightforward to see that if $\tilde{c} \neq 0$ no dS solution with $H=$ const is allowed, not even with a time-varying

\footnotetext{
${ }^{6}$ See also [50] for a study on the compatibility between swampland conjectures and asymptotically safe gravity.

${ }^{7}$ More precisely, $\Lambda \propto \frac{D_{\text {crit }}-D}{\alpha^{\prime}}$ encodes the deviation from the critical dimension, namely, $D_{\text {crit }}=26,10$ for bosonic strings and superstrings, respectively $[28,51,52]$.
} 
dilaton $\phi$. If instead $\tilde{c}=0$, the above cosmological equations admit a unique solution with constant $H$ and

$$
\Phi(t)=C \pm \sqrt{\Lambda} t
$$

$C$ being an integration constant. However, this quasi-dS solution is only realized in an unphysical regime since $\tilde{c}=0$ entails trivial gravitational dynamics at the level of the effective action $\Gamma_{\text {string }}$. Let us remark that the solution above, while exact, might not be unique. A proper analysis of the flow equations, including the space of initial conditions compatible with UV fixed points, appears prohibitive in general, but, as we shall now discuss, it is feasible in $2+\epsilon$ dimensions.

The effective action of Eq. (9) is neither analytic in the curvatures (thus, it is unable to reproduce string theory in the UV or general relativity in the IR) nor expected to be unique. We are therefore led to seek analytic, and possibly general, solutions to the RG equations. To this end, it is useful to employ an $\epsilon$-expansion of the effective action about $D=2$, along the lines of [2,53-59], and then try to extend the results to $D=4$. The rationale behind this approach rests on the observation that the Newton coupling is classically marginal in $D=2$ dimensions, and therefore, the RG flow dramatically simplifies expanding $D=2+\epsilon$ for $\epsilon \ll 1$. Since one expects a UV fixed point for $g_{k}$ of order $\mathcal{O}(\epsilon)$, our starting point is the ansatz $g_{k}=\epsilon \gamma_{k}$, with $\gamma_{k}=\mathcal{O}(1)$, while the ansatz

$$
F_{k}\left(H^{2}\right)=\frac{v_{k}\left(H^{2}\right)}{\epsilon \gamma_{k}}+w_{k}\left(H^{2}\right),
$$

with $v_{k}\left(H^{2}\right), w_{k}\left(H^{2}\right)=\mathcal{O}(1)$, is motivated by the expected IR behavior of $F_{k}$, whose corrections ought to be subleading in $\epsilon$. The resulting flow equations simplify dramatically at leading order in $\epsilon$. As a result, the dimensionless Newton coupling flows according to $\gamma_{k}=\gamma_{*}\left(1+c k^{-\epsilon}\right)^{-1}$, with $c$ a constant. This flow mirrors that of Eq. (7) for $d=1+\epsilon$, and in particular, it starts from $\gamma_{*}=\frac{3}{2} \pi^{2}$ in the UV and ends at zero in the IR for $c>0$, where $\gamma_{k} \sim \frac{\gamma_{*}}{c} k^{\epsilon}$. The corresponding IR value of the dimensionful Newton coupling is then $G_{k} \sim \frac{3 \pi}{32} \frac{\epsilon}{c}$. The next step involves the subleading corrections, where $\dot{\gamma_{k}} \sim \epsilon \gamma_{k}$ can be neglected with respect to the other contributions. The subleading flow equations can be solved in closed form, and in the IR, one obtains higher-derivative corrections to the low-energy effective action to all orders in $H$, and thus in $\alpha^{\prime}$, since the $k$ dependence correctly disappears.

The most general analytic solution to the exact flow equation in $2+\epsilon$ dimensions compatible with a UV fixed point leads to the $\mathcal{O}(\epsilon)$ effective action [32]

$$
\begin{aligned}
\Gamma_{\text {string }}= & \frac{\mathrm{Vol}_{1+\epsilon}}{16 \pi G_{\mathrm{N}}} \int d t n e^{-\Phi} \\
& \times\left[\Lambda-\frac{\dot{\Phi}^{2}}{n^{2}}+\frac{H^{2}}{n^{2}}+\frac{8 G_{\mathrm{N}} \Lambda}{3 \pi} L\left(\frac{H^{2}}{n^{2} \Lambda}\right)\right],
\end{aligned}
$$

where the relevant deformation $\Lambda$ from the UV fixed point controls the validity of the low-energy expansion, and

$$
\begin{aligned}
L(s) \equiv & -1-\frac{23}{12} s+\left(\frac{3}{2}+s\right) \log \left(1+\frac{s}{2}\right) \\
& +(1+s)^{\frac{3}{2}} \sqrt{\frac{2}{s}} \operatorname{arctanh}\left(\sqrt{\frac{s}{2(1+s)}}\right)
\end{aligned}
$$

is depicted in Fig. 2. As expected, quadratic corrections to the classical action dominate at low curvatures $(H \ll \Lambda)$. The effective cosmological equations in $(2+\epsilon)$ dimensions read

$$
\begin{aligned}
\dot{\Phi}^{2} & =H^{2}\left[1+\frac{16 G}{3 \pi} L^{\prime}\left(\frac{H^{2}}{\Lambda}\right)\right]-\Lambda\left[1+\frac{8 G}{3 \pi} L\left(\frac{H^{2}}{\Lambda}\right)\right], \\
\ddot{\Phi} & =H^{2}\left[1+\frac{8 G}{3 \pi} L^{\prime}\left(\frac{H^{2}}{\Lambda}\right)\right], \\
\frac{\dot{H}}{H} & =\dot{\Phi}-\frac{8 G}{3 \pi} L^{\prime}\left(\frac{H^{2}}{\Lambda}\right)\left(\frac{\dot{H}}{H}-\dot{\Phi}\right)-\frac{16 G H \dot{H}}{3 \pi \Lambda} L^{\prime \prime}\left(\frac{H^{2}}{\Lambda}\right),
\end{aligned}
$$

where we have omitted the dependence on $t$ for brevity. Specializing Eq. (15) to the case of constant $H$ yields the existence conditions for nonperturbative dS solutions discussed in $[33,60]$. It is straightforward to see that $\mathrm{dS}$ solutions are not allowed by Eq. (15) since the conditions of

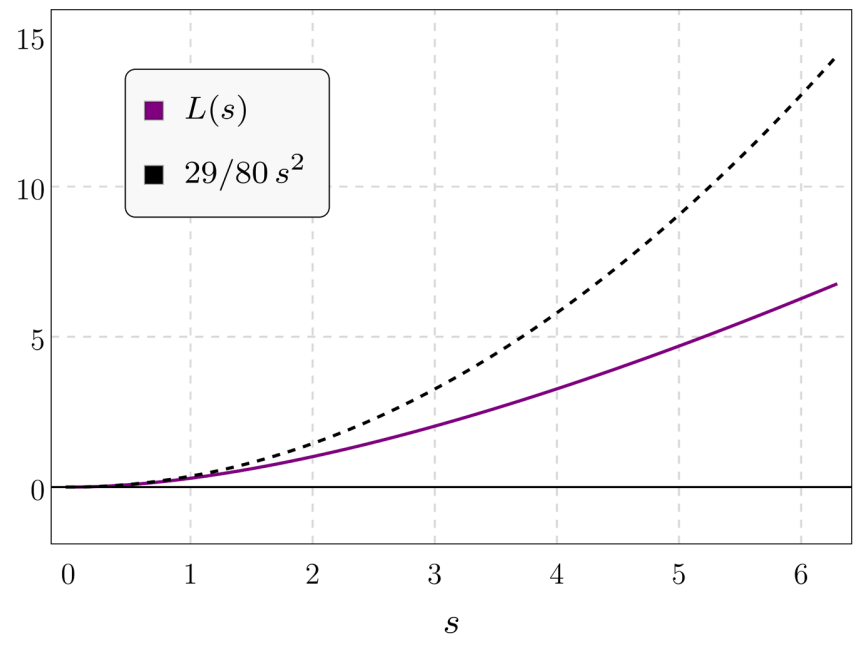

FIG. 2. Function $L(s)$ (purple curve) encoding higherderivative $\alpha^{\prime}$ corrections to the classical action. As expected, terms proportional to $s^{2} \sim R^{2}$ (black dashed line) dominate the $\alpha^{\prime}$-expansion of the effective action at low curvatures. 
[33] cannot be met by the all-order $\alpha^{\prime}$-corrections encoded in the (monotone increasing) $L(s)$ of Eq. (14). Even in the Einstein frame, the Hubble parameter satisfies [33]

$$
H_{E} \stackrel{\epsilon \rightarrow 0}{\sim} \frac{d}{d t} e^{\frac{1}{\epsilon}(\sigma+\Phi)},
$$

which entails $H \sim-\dot{\Phi}$ for an Einstein-frame dS solution. This is also nontrivially incompatible with Eq. (15). Even in the Einstein frame, the Hubble parameter satisfies [33]

$$
H_{E} \stackrel{\epsilon \rightarrow 0}{\sim} \frac{d}{d t} e^{\frac{1}{\epsilon}(\sigma+\Phi)},
$$

which entails $H \sim-\dot{\Phi}$ for an Einstein-frame dS solution. This is also nontrivially incompatible with Eq. (15). Analogously, the nonanalytic effective action in Eq. (9) does not allow dS solutions. Moreover, at least within polynomial truncations up to order $\mathcal{O}\left(H^{10}\right)$, the effective action in Eq. (13) does not extend to $D=4$ [32]. We are thus led to conclude that, in this setting, insofar as only minisuperspace cosmologies are included in the gravitational functional integral, our results point to a tension between $\mathrm{dS}$ solutions and curvature corrections in string theory. Our findings thus support the no-dS swampland conjecture [36-38] in the presence of all-order effects in $\alpha^{\prime}$.

Let us finally remark that, although a highly curved dS spacetime cannot be described at the level of two-derivative (super)gravity, it could have, in principle, arisen from our infinite-derivative field equations. In this case, the resulting cosmological constant would have most likely been of the order of the string scale.

\section{CONCLUSIONS}

Understanding higher-derivative corrections and all-order effects appears of fundamental importance for high-energy regimes of gravity. In particular, they could play a crucial role in the resolution of singularities and in the structure of the fundamental degrees of freedom and their interactions. Moreover, they could be crucial to assess the existence of dS solutions, in particular, in the context of string theory. In this respect, while string theory provides, at least in some regimes, well-defined algorithms to systematically compute such corrections, earlier attempts to derive them in the general case were met by intricate technical difficulties. Therefore, we are compelled to investigate novel directions in order to shed light on these relevant matters.

To this end, symmetries play a key role. Indeed, although direct computations of all-order effects are extremely involved in general, stringy symmetries such as T-duality constrain their form substantially [29-31,33,34], thus allowing us to investigate the existence of $\mathrm{dS}$ solutions in string theory beyond tree level. Earlier works in this direction [61-63] involved the leading curvature corrections to the classical action and discovered that-to leading order in
$\alpha^{\prime}-\mathrm{dS}$ vacua are excluded by the simultaneous presence of the dilaton and $\alpha^{\prime}$-corrections [61,62].

In this work we have taken a major step forward by resumming all $\alpha^{\prime}$ corrections on cosmological backgrounds. Specifically, combining the constraints on stringy cosmological effective actions with FRG techniques [32], in this paper we have derived the cosmological field equations associated with two closed-form $\alpha^{\prime}$-corrected effective actions, and we have investigated the existence of $\mathrm{dS}$ solutions in the presence of all-order $\alpha^{\prime}$-corrections. No $\mathrm{dS}$ solution seems to be allowed. Our findings thus support the implications of the relevant swampland conjectures [36-38] in the presence of curvature corrections to all orders in $\alpha^{\prime}$.

All in all, within the framework of string theory, our results appear to point at one (or more) of the following possibilities:

(i) The existence of dS solutions in string theory requires inhomogeneities and/or anisotropies, e.g., to generalize the minisuperspace ansatz to Bianchilike models.

(ii) dS solutions require the inclusion of string-loop corrections.

(iii) No dS solution is allowed in string theory, and early/ late-time phases of accelerated expansion are driven by quintessence (or analogous scenarios) [64-66].

Comparing the FRG approach presented in this work with the results of perturbative string theory in its critical dimension remains an important, if daunting, undertaking. As pointed out in [32], it is currently challenging to test our formalism due to the considerable difficulty of solving the FRG equations in sufficient generality in higher dimensions. The only setting in which this is currently feasible is within an epsilon-expansion about $D=2$, where we managed to obtain the most general flow compatible with a UV fixed point. Comparing our results to bona fide string perturbation theory computations would require, at least, resumming this expansion or finding general UV-complete surfaces in higher dimensions. Alternatively, one would need to compute the $\alpha^{\prime}$-exact effective action in noncritical dimensions in string theory, which is currently out of reach (except for special—noncosmological—backgrounds).

At the same time, while difficult to test, the interplay between the FRG and the symmetries of string theory could open new doors, allowing us to investigate important nonperturbative aspects of phenomenologically relevant scenarios otherwise unaccessible with standard perturbative methods. In particular, considerations similar to those that we have put forth in this paper, as well as in [32], could be applied to a number of scenarios where allorder effects are expected to be important, for instance, the cancellation of Weyl anomalies of the string world sheet [67], thereby providing a new and rich avenue of research. We would like to explore these intriguing ideas and scenarios in future work. 


\section{ACKNOWLEDGMENTS}

The authors would like to thank A. Bonanno, A. Eichhorn, B. Knorr, A. Sagnotti, and F. Saueressig for discussions. A. P. acknowledges support by Perimeter Institute for Theoretical Physics. Research at Perimeter Institute is supported in part by the Government of Canada through the Department of Innovation, Science and Economic Development Canada and by the Province of Ontario through the Ministry of Colleges and Universities. The work of I. B. was supported by the Fonds de la Recherche Scientifique-FNRS under Grants No. F.4503.20 ("HighSpinSymm") and No. T.0022.19 ("Fundamental issues in extended gravitational theories").
[1] S. Weinberg, Critical phenomena for field theorists, Erice Subnuclear Physics (1976).

[2] S. Weinberg, Ultraviolet divergences in quantum theories of gravitation, in General Relativity: An Einstein centenary survey, edited by S. W. Hawking and W. Israel (1979), pp. 790-831.

[3] W. Souma, Nontrivial ultraviolet fixed point in quantum gravity, Prog. Theor. Phys. 102, 181 (1999).

[4] A. Bonanno, A. Eichhorn, H. Gies, J. M. Pawlowski, R. Percacci, M. Reuter, F. Saueressig, and G. P. Vacca, Critical reflections on asymptotically safe gravity, Front. Phys. 8, 269 (2020).

[5] D. Amati and C. Klimcik, Strings in a shock wave background and generation of curved geometry from flat space string theory, Phys. Lett. B 210, 92 (1988).

[6] D. Amati and C. Klimcik, Nonperturbative computation of the Weyl anomaly for a class of nontrivial backgrounds, Phys. Lett. B 219, 443 (1989).

[7] S. Kachru, J. Pearson, and H. L. Verlinde, Brane/flux annihilation and the string dual of a nonsupersymmetric field theory, J. High Energy Phys. 06 (2002) 021.

[8] S. Kachru, R. Kallosh, A. D. Linde, and S. P. Trivedi, de Sitter vacua in string theory, Phys. Rev. D 68, 046005 (2003).

[9] D. Kutasov, T. Maxfield, I. Melnikov, and S. Sethi, Constraining de Sitter Space in String Theory, Phys. Rev. Lett. 115, 071305 (2015).

[10] U. H. Danielsson and T. Van Riet, What if string theory has no de Sitter vacua?, Int. J. Mod. Phys. D 27, 1830007 (2018).

[11] M. Cicoli, S. De Alwis, A. Maharana, F. Muia, and F. Quevedo, De Sitter vs quintessence in string theory, Fortschr. Phys. 67, 1800079 (2019).

[12] F. Gautason, V. Van Hemelryck, and T. Van Riet, The tension between 10D supergravity and dS uplifts, Fortschr. Phys. 67, 1800091 (2019).

[13] Y. Hamada, A. Hebecker, G. Shiu, and P. Soler, Understanding KKLT from a 10d perspective, J. High Energy Phys. 06 (2019) 019.

[14] F. Gautason, V. Van Hemelryck, T. Van Riet, and G. Venken, A 10d view on the KKLT AdS vacuum and uplifting, J. High Energy Phys. 06 (2020) 074.

[15] K. Dasgupta, M. Emelin, M. M. Faruk, and R. Tatar, de Sitter vacua in the string landscape, Nucl. Phys. B969, 115463 (2021).
[16] E. Dudas and J. Mourad, Brane solutions in strings with broken supersymmetry and dilaton tadpoles, Phys. Lett. B 486, 172 (2000).

[17] N. Kitazawa and A. Sagnotti, String theory clues for the low- $\ell$ CMB?, EPJ Web Conf. 95, 03031 (2015).

[18] N. Kitazawa and A. Sagnotti, A string-inspired model for the low- $\ell$ CMB, Mod. Phys. Lett. A 30, 1550137 (2015).

[19] A. Sagnotti, Low- $\ell$ CMB from string-scale SUSY breaking?, Mod. Phys. Lett. A 32, 1730001 (2017).

[20] I. Basile and S. Lanza, de Sitter in non-supersymmetric string theories: No-go theorems and brane-worlds, J. High Energy Phys. 10 (2020) 108.

[21] N. Dupuis, L. Canet, A. Eichhorn, W. Metzner, J. Pawlowski, M. Tissier, and N. Wschebor, The nonperturbative functional renormalization group and its applications, Phys. Rep. 910, 1 (2021).

[22] C. Wetterich, Exact evolution equation for the effective potential, Phys. Lett. B 301, 90 (1993).

[23] M. Bojowald, Absence of a Singularity in Loop Quantum Cosmology, Phys. Rev. Lett. 86, 5227 (2001).

[24] J. Feldbrugge, J.-L. Lehners, and N. Turok, No Smooth Beginning for Spacetime, Phys. Rev. Lett. 119, 171301 (2017).

[25] J. B. Hartle, S. W. Hawking, and T. Hertog, No-Boundary Measure of the Universe, Phys. Rev. Lett. 100, 201301 (2008).

[26] J. D. Dorronsoro, J. J. Halliwell, J. B. Hartle, T. Hertog, O. Janssen, and Y. Vreys, Damped Perturbations in the No-Boundary State, Phys. Rev. Lett. 121, 081302 (2018).

[27] A. Ashtekar, T. Pawlowski, and P. Singh, Quantum Nature of the Big Bang, Phys. Rev. Lett. 96, 141301 (2006).

[28] G. Veneziano, Scale factor duality for classical and quantum strings, Phys. Lett. B 265, 287 (1991).

[29] K. Meissner and G. Veneziano, Symmetries of cosmological superstring vacua, Phys. Lett. B 267, 33 (1991).

[30] K. A. Meissner, Symmetries of higher order string gravity actions, Phys. Lett. B 392, 298 (1997).

[31] O. Hohm and B. Zwiebach, T-duality constraints on higher derivatives revisited, J. High Energy Phys. 04 (2016) 101.

[32] I. Basile and A. Platania, Cosmological $\alpha^{\prime}$-corrections from the functional renormalization group, J. High Energy Phys. 06 (2021) 045.

[33] O. Hohm and B. Zwiebach, Non-perturbative de Sitter vacua via $\alpha^{\prime}$ corrections, Int. J. Mod. Phys. D 28, 1943002 (2019). 
[34] O. Hohm and B. Zwiebach, Duality invariant cosmology to all orders in $\alpha^{\prime}$, Phys. Rev. D 100, 126011 (2019).

[35] E. Palti, The swampland: Introduction and review, Fortschr. Phys. 67, 1900037 (2019).

[36] G. Obied, H. Ooguri, L. Spodyneiko, and C. Vafa, De Sitter space and the swampland, arXiv:1806.08362.

[37] H. Ooguri, E. Palti, G. Shiu, and C. Vafa, Distance and de Sitter conjectures on the swampland, Phys. Lett. B 788, 180 (2019).

[38] S. K. Garg and C. Krishnan, Bounds on slow roll and the de Sitter swampland, J. High Energy Phys. 11 (2019) 075.

[39] S. W. Hawking, Quantum cosmology, in Les Houches Summer School on Theoretical Physics: Relativity, Groups and Topology (1983), pp. 333-379.

[40] S. W. Hawking, The quantum state of the universe, Nucl. Phys. B239, 257 (1984).

[41] A. Vilenkin, Approaches to quantum cosmology, Phys. Rev. D 50, 2581 (1994).

[42] A. Ashtekar and P. Singh, Loop quantum cosmology: A status report, Classical Quant. Grav. 28, 213001 (2011).

[43] M. Bojowald, Quantum cosmology: A review, Rep. Prog. Phys. 78, 023901 (2015).

[44] T. R. Morris, The exact renormalization group and approximate solutions, Int. J. Mod. Phys. A 09, 2411 (1994).

[45] M. Reuter, Nonperturbative evolution equation for quantum gravity, Phys. Rev. D 57, 971 (1998).

[46] B. Knorr, C. Ripken, and F. Saueressig, Form factors in asymptotic safety: Conceptual ideas and computational toolbox, Classical Quant. Grav. 36, 234001 (2019).

[47] A. Bonanno and M. Reuter, Renormalization group improved black hole space-times, Phys. Rev. D 62, 043008 (2000).

[48] S. de Alwis, A. Eichhorn, A. Held, J. M. Pawlowski, M. Schiffer, and F. Versteegen, Asymptotic safety, string theory and the weak gravity conjecture, Phys. Lett. B 798, 134991 (2019).

[49] S. Weinberg, On the development of effective field theory, Eur. Phys. J. H 46, 6 (2021).

[50] I. Basile and A. Platania, Asymptotic safety: Swampland or wonderland?, Universe 7, 389 (2021).

[51] E. S. Fradkin and A. A. Tseytlin, Effective field theory from quantized strings, Phys. Lett. 158B, 316 (1985).

[52] J. Callan, G. Curtis, E. Martinec, M. Perry, and D. Friedan, Strings in background fields, Nucl. Phys. B262, 593 (1985).
[53] R. Gastmans, R. Kallosh, and C. Truffin, Quantum gravity near two-dimensions, Nucl. Phys. B133, 417 (1978).

[54] S. Christensen and M. Duff, Quantum gravity in two $+\epsilon$ dimensions, Phys. Lett. 79B, 213 (1978).

[55] H. Kawai and M. Ninomiya, Renormalization group and quantum gravity, Nucl. Phys. B336, 115 (1990).

[56] H. Kawai, Y. Kitazawa, and M. Ninomiya, Scaling exponents in quantum gravity near two-dimensions, Nucl. Phys. B393, 280 (1993).

[57] H. Kawai, Y. Kitazawa, and M. Ninomiya, Ultraviolet stable fixed point and scaling relations in $(2+\epsilon)$-dimensional quantum gravity, Nucl. Phys. B404, 684 (1993).

[58] H. Kawai, Y. Kitazawa, and M. Ninomiya, Renormalizability of quantum gravity near two-dimensions, Nucl. Phys. B467, 313 (1996).

[59] T. Aida and Y. Kitazawa, Two loop prediction for scaling exponents in $(2+\epsilon)$-dimensional quantum gravity, Nucl. Phys. B491, 427 (1997).

[60] C. A. Núñez and F. E. Rost, New non-perturbative de Sitter vacua in $\alpha^{\prime}$-complete cosmology, J. High Energy Phys. 03 (2021) 007.

[61] D. G. Boulware and S. Deser, Effective gravity theories with dilatons, Phys. Lett. 175B, 409 (1986).

[62] M. C. Bento and O. Bertolami, Maximally symmetric cosmological solutions of higher curvature string effective theories with dilatons, Phys. Lett. B 368, 198 (1996).

[63] A. L. Maroto and I. L. Shapiro, On the inflationary solutions in higher derivative gravity with dilaton field, Phys. Lett. B 414, 34 (1997).

[64] P. Agrawal, G. Obied, and C. Vafa, $H_{0}$ tension, swampland conjectures, and the epoch of fading dark matter, Phys. Rev. D 103, 043523 (2021).

[65] A. Banerjee, H. Cai, L. Heisenberg, E. O. Colgáin, M. M. Sheikh-Jabbari, and T. Yang, Hubble sinks in the low-redshift swampland, Phys. Rev. D 103, 081305 (2021).

[66] E. Di Valentino, O. Mena, S. Pan, L. Visinelli, W. Yang, A. Melchiorri, D. F. Mota, A. G. Riess, and J. Silk, In the realm of the Hubble tension: A review of solutions, Classical Quant. Grav. 38, 153001 (2021).

[67] I. Basile and A. Platania, Worldsheet anomaly cancellation, functional renormalization and all-order $\alpha^{\prime}$ corrections (to be published). 